J. Natn. Sci. Coun. Sri Lanka 1987 15(1) : 83-86

\title{
ON SOME SEQUENCE TO SEQUENCE TRANSFORMATIONS
}

\section{YOGACHANDRAN}

Department of Matbematics and Statistics, University of Maiduguri, Nigeria.

(Date of receipt : 23 December 1985)

(Date of acceptance : 24 March 1987)

Abstract : In this paper we prove two theorems on sequence to sequence transformations given by $y_{\mathrm{n}}=\sum_{\text {rel }}^{\infty} \mathrm{a}_{\mathrm{nk}} \mathrm{x}_{\mathrm{k}}, \mathrm{n}=1,2, \ldots$ The theorems give necessary and
sufficient conditions in order that

(1) $y \in l$ whenever $x \in b v$, and
(2) $y \in b v$ whenever $x \in 1$, where $x=\left\{x_{n}\right\}$ and $y=\{y\}$, and $I=$ The space of all sequences $x$ such that $\sum_{n=1}^{\infty}\left|x_{n}\right|<\infty, 1^{\infty}=$ The space of al sequences $x$ such that $x_{n}=0(1)$, bv $=$ The space of all $x$ such that $\sum_{n=1}^{\infty}\left|x_{n}-x_{n-1}\right|<\infty$.

\section{Introduction}

We assume that the transformation is given by

$$
y_{n}=\sum_{n=1}^{\infty} a_{n k} x_{k}, n=1,2,3, \ldots
$$

We prove the following theorems:

1.1 THEOREM 1 : Necessary and sufficient conditions in order that $y \in 1$ whenever $\mathrm{x} \in \mathrm{bv}$ are :
(i) $\sum_{\mathrm{k}=1}^{\infty} a_{\mathrm{nk}}$ converges for every $\mathrm{n}=1,2 \ldots \ldots$
(ii) There exists $M>0$ such that $\sum_{n=1}^{\infty}\left|\sum_{k=1}^{m} a_{n k}\right| \leqslant M$ for all $m \geqslant 1$.

1.2 THEOREM 2 : Necessary and sufficient conditions in order that $y \in$ by whenever $\mathrm{x} \in 1^{\infty}$ are that

(i) $2 \sum_{k=1}^{\infty}\left|a_{n k}\right|<\infty$ for every $n \geqslant 1$.

(ii) $)_{2}$ There exists $M>0$ such that $\sum_{k=1}^{\infty}\left|\sum_{n \in N}\left(a_{n k}-a_{n-1, k}\right)\right| \leqslant M$

for all $N \in F$, where $F$ is the space of all finite sets $N$ of natural numbers.

There are many well known classical theorems on sequence to sequence transformations of the above type, some of which have been proved by Toeplitz, Hahn, Knopp and Lorentz, Mears, Sunouchi and others. Usually, the longest part of the proof is the proof of the necessity of conditions of 
the type (ii) ${ }_{1}$ and (ii) ${ }_{2}$. In this paper, we give a technique which shortens the proof considerably. (See (1) for similar theorems and for the references given. See also (2) for the use of this technique for integral transformations.)

\section{Results used in the Proofs of the Theorems}

\subsection{THEOREM A (Toeplitz's Theorem)} $\mathrm{x} \in 1^{\infty}$ are :

Necessary and sufficient conditions in order that $y \in 1^{\infty}$ whenever

(i) $\sum_{k=1}^{\infty}\left|a_{n k}\right|<\infty$ for every $n \geqslant 1$

(ii). There exists $M>0$ such that $\cdot \sum_{\mathrm{k}=1}^{\infty}\left|a_{\mathrm{nk}}\right| \leqslant M$ for all $n \geqslant 1$.

2.2 THEOREM B (Hahn's Theorem)

Necessary and sufficient conditions in order that $y \in 1^{\infty}$ whenever $\mathrm{x} \in \mathrm{bv}$ are that $n \geqslant 1$.

(i) $\sum_{\mathrm{k}=1}^{\infty} a_{\mathrm{n} k}$ converges for every $\mathrm{n} \geqslant 1$

(ii) $b_{b}$ There exists $M>0$ such that $\left|\sum_{k=1}^{m}, a_{n k}\right| \leqslant M$ for all $m \geqslant 1$ and all

These two theorems are well known.

The following lemma is a simple consequence of the definition of absolute convergence. (See (1).

LEMMA 1: Let $\mathrm{F}$ be the space of all finite sets of natural numbers. Then, a necessary and sufficient condition that $\mathrm{x} \in 1$ is that :

I. There exists $K$ independent of $N$ such that $\left|B_{N}\right| \leqslant K$ for all $N \in F$, where $B_{N}=\sum_{n} x_{N}$.

When this condition is satisfied, we have $\sum_{n=1}^{\infty}\left|x_{n}\right| \leqslant 2 K$.

LEMMA 2: Let $\mathrm{y}_{\mathrm{n}}=\sum_{\mathrm{k}=1}^{\infty} \mathrm{a}_{\mathrm{n}} \mathrm{x}_{\mathrm{k}}$, where $\mathrm{x} \in \mathrm{X}, \mathrm{X}$ being a sequence space.
Then,

I. $\mathrm{y} \in \mathrm{l}$ for every $\mathrm{x} \in \mathrm{X}$ iff $\mathrm{B}_{\mathrm{N}}$ is bounded for all $\mathrm{N} \in \mathrm{F}$ for every $\mathrm{x} \in \mathrm{X}$, where $B_{N}=\sum_{k=1}^{\infty} b_{N, k} x_{k}$ and $b_{N, k}=\sum_{n \in N} a_{n k}$. 
II. $y \in$ bv for every $x \in X$ if $D_{N}$ is bounded for all $N \in F$ for every $\mathrm{x} \in \mathrm{X}$, where $\mathrm{D}_{\mathrm{N}}=\sum_{\mathrm{k}=1}^{\infty} \mathrm{d}_{\mathrm{N}, \mathrm{k}} \mathrm{x}_{\mathrm{k} .}$ and $\mathrm{d}_{\mathrm{N}, \mathrm{k}} \doteq \sum_{\mathrm{n} \in \mathrm{N}}\left(\mathrm{a}_{\mathrm{nk}}-\mathrm{a}_{\mathrm{n}-1, \mathrm{k}}\right)$.

Proof I : By Lemma $1, y \in 1$ iff $\left|B_{N}\right| \leqslant K$ for all $N \in F$, where $B_{N}=\sum_{n \in N} y_{n}$. But $B_{N}=\sum_{n \in N} \sum_{k=1}^{\infty} a_{n k} x_{k}=\sum_{k=1}^{\infty}\left(\sum_{n \in N} a_{n k}\right) x_{k}=\sum_{k=1}^{\infty} b_{N, k} x_{k}$.

Hence the result.

II. $y \in$ bv iff $\sum_{n=1}^{\infty}\left|y_{n}-y_{n-1}\right|<\infty$
Since $y_{n}-y_{n-1}=\sum_{k=1}^{\infty}\left(a_{n k}-a_{n-1, k}\right) x_{k}$, the result follows.

\section{Proofs of the Theorems}

3.1 Proof of Theorem 1: Note that $(i)_{1}$ is necessary and sufficient for the existence of $y_{n}$ whenever $x \in b v$. (Theorem $B$ ).

Sufficiency: If (i) ${ }_{1}$ and (ii) ${ }_{1}$ hold and $\mathrm{x} \in \mathrm{bv}$, then

$\left|\sum_{k=1 \times}^{m} b_{N, k}\right|=\left|\sum_{k=1}^{m} \sum_{n \in N} a_{n k}\right|=\left|\sum_{n \in N} \sum_{k=1}^{n i} a_{n k}\right| \leqslant \sum_{n=1}^{\infty}\left|\sum_{k=1}^{m} a_{n k}\right| \leqslant M$ for all $N \in F$,

By Theorem $B$, this implies that $\sum_{k=1}^{\infty} b_{N}, x_{k}$ is bounded for all $N \in$ F. Hence
by Lemma $2, y \in 1$.

Necessity By Lemma 2, $y \in 1$ implies that $B_{N}=0(1)$ for all $N \in F$ for every $\mathrm{x} \in \mathrm{bv}$.

Hence, by Theorem B, there exists $M$ such that

$1 \sum_{\mathrm{k}=1}^{\mathrm{m}}$

$\mathrm{b}_{\mathrm{N}, \mathrm{k}} \mid \leqslant 1 / 2 \mathrm{M}$ for all $\mathrm{N} \in \mathrm{F}$ and all $\mathrm{m}$.

i.e. $\left|\sum_{n \in N} \sum_{k=1}^{m} a_{n k}\right| \leqslant 1 / 2 M$ for all $N \in F$ and all $m$, and Lemma 1 gives the required condition $(\mathrm{ii})_{1}$.

Hence the theorem.

3.2 Proof of Theorem 2: (i) 2 is necessary and sufficient for the existence of $\mathrm{y}_{\mathrm{n}}$ whenever $\mathrm{x} \in 1^{\infty}$. (Theorem $\mathrm{A}$ ).

Sufficiency: If (i) ${ }_{2}$ and (ii) ${ }_{2}$ hold, and $x \in 1^{\infty}$, then

$\left|D_{N}\right|=\left|\sum_{k=1}^{\infty} \sum_{n \in N}\left(a_{n k}-a_{n-1, k}\right) x_{k}\right| \leqslant M \sum_{k=1}^{\infty}\left|x_{k}\right|$ and hence $y \in$ bv by

Lemma 2 II. 
Necessity: Again by Lemma 2 II, $y \in$ bv implies that

$$
\mathrm{D}_{\mathrm{N}}=\sum_{\mathrm{k}=1}^{\infty} \mathrm{d}_{\mathrm{N}, \mathrm{k}} \mathrm{x}_{\mathrm{k}} \text { is bounded for all } \mathrm{N} \in \mathrm{F} \text { whenever } \mathrm{x} \in 1^{\infty} \text {, and }
$$

Theorem A gives the necessary condition

$$
\sum_{k=1}^{\infty}\left|d_{N, k}\right| \leqslant M \text { for all } N \in F \text {, which is }(i i)_{2} .
$$

Note: We see that condition (ii) ${ }_{2}$ involves $\mathrm{N}$. We can show that (ii) ${ }_{2}$ cannot be replaced by the condition:

(ii)' $: \sum_{k=1}^{\infty} \sum_{n=1}^{\infty}\left|a_{n k}-a_{n-1, k}\right|<\infty$, which is independent of N. Clearly, (i) and (ii)' are sufficient for $y$ to be in bv whenever $x \in 1^{\infty}$, but (ii)' is not necessary.

In (1), Peyerimhoff has constructed $x \in 1^{\infty}$ for which $y_{n}^{\prime}=\sum_{k=1}^{\infty} a_{n k}^{\prime} x_{k}$ satisfies $\sum_{n=1}^{\infty} T y_{n}^{\prime} T_{<\infty}$ but $\sum_{k=1}^{\infty} \sum_{n=1}^{\infty} l a_{n k}=L$.

By taking $a_{n k}=a_{n k}-a_{n-1, k}, y_{n}=\sum_{k=1}^{\infty} a_{n k} x_{k}$, we see that for the same $x$ in $1^{\infty}, \sum_{n=1}^{\infty}\left|y_{n}-y_{n-1}\right|<\infty$, but

$$
\sum_{k=1}^{\infty} \sum_{n=1}^{\infty}\left|a_{n k}-a_{n-1, k}\right|=\infty \text {. }
$$

Thus (ii)' is not necessary in Theorem 2 .

\section{References:}

1. PEYERIMHOFF, A. (1957) Journal London Math. Soc. 32 : 33-36.'

2. YOGACHANDRAN, C. (1973) Journal London Math. Soc, $216: 639-648$. 